\title{
Integrated planning and scheduling for multi-product job-shop assembly based on genetic algorithms
}

\author{
M. F. Sebaaly and H. Fujimoto \\ Department of Mechanical Engineering, Nagoya Institute of \\ Technology, Gokiso-cho, Showa-ku, Nagoya 466, JAPAN, \\ Tel: +81-52-735-5330 Fax: +81-52-735-5342 \\ E-mail:milad@vier.mech.nitech.ac.jp
}

\begin{abstract}
This paper introduces a simultaneous approach for dealing with assembly sequence planning and scheduling, which have been dealt with separately in research. Assembly planning consists of finding the optimal or best sequence to assemble a certain product, according to some product criteria. Assembly scheduling is concerned with finding the optimal or best schedule to perform the assembly operations by a given number of machines, according to some system criteria such as time-in-process(TIP) and idle time. However, a best sequence necessarily leads to an efficient operations schedule, and a best schedule might alter the feasibility precedence constraints of the assembly sequence. These cases can be best encountered in multi-product job-shop assembly. This paper introduces a genetic algorithm approach to integrate the two procedures together. A prototype example is solved to illustrate the new approach.
\end{abstract}

\section{Keywords}

Flexible assembly, planning and scheduling, genetic algorithms, concurrent engineering. 


\section{INTRODUCTION}

This paper addresses a multi-product flexible assembly problem in a job-shop environment. The considered flexible assembly system (FAS) consists of a number of flexible assembly cells or machines, each having the capabilities of performing a family of assembly jobs or operations. For every product at hand, two questions need to be answered in order to implement an optimal or best assembly process resulting in competitive products. First, what is the best sequence to assemble the product part according to various product criteria such as stability, manipulability, directionality and cost (Pu, 1992; Wolter, et al., 1992). Second, what is the best schedule assigning appropriate jobs for each machine to assemble the product, according to several scheduling criteria such as product time-in-process (TIP) and machine idle time (Fujimoto, et al., 1994; Lucertini, et al., 1994). Until recently, these questions have been dealt with separately in research, and classified as assembly planning and scheduling. Apparently, this separation resulted in partial independent results that are unaware of the mutual dependence and interaction. In assembly planning for instance, the optimal or best sequences obtained to assemble a certain product do not necessarily take into consideration the cascading of assembly operations and jobs needed every time a part is assembled, and the effect of this cascading on the system balance, the machines idle times and the product TIP. On the other hand, the optimal or best schedules obtained to assemble a certain product, with the best TIP, idle times and/or other scheduling criteria, do not necessarily take into consideration the product stability, manipulability, directionality, etc. Consequently, an optimal parts sequence best satisfying the planning criteria might lead to bottlenecks resulting in longer machine idle times and product waiting times. Similarly, an optimal job schedule best satisfying the scheduling criteria might result in difficult manipulability conditions, unstable or marginally stable subassemblies, or even invalid assembly sequences. Both outputs result in assembly process difficulties producing less competitive products. This problem is frequently encountered in a multi-product job-shop environment.

This paper addresses the problem of integrated assembly planning and scheduling. The objective of this work is to simultaneously generate the best sequences to assemble a group of products and the best schedule of corresponding jobs. The adopted methodology is rather an extension of the work in (Sebaaly and Fujimoto, 1996) that deals with automatic assembly planning based on Genetic Algorithms. A best assembly sequence is generated without searching the whole solution space. Search decisions are made for the entire assembly sequence rather than on a part-by-part basis. This paper extends this methodology in two dimensions: Several products are considered at the same time, and both planning and scheduling criteria are represented in the objective function. The considered environment consists of a fixed number of flexible assembly cells connected to each other by flexible handling machines or robots. The GA is applied to a population of 
individuals representing both assembly sequences and schedules. The fitness function considered by the GA selection operator is a multiobjective function linearly combining the planning and scheduling criteria with random weights. The proposed algorithm is applied to a prototype example, where its various steps are illustrated.

\section{ASSEMBLY SEQUENCE PLANNING}

The assembly sequence planning (ASP) problem can be simply stated as how to find an optimal or best sequence to assemble a given product, given its $\mathrm{CAD}$ design files. Various optimisation criteria are considered, which are related to the internal dynamics of the assembly operations, such as parts manipulability, operations directionality, and tools cost. Several planners were introduced in research to tackle this problem, most of which share one or more of the following difficulties (Pu,1992; Wolter, et al., 1992): a- searching the complete solution space (exact solution). b- performing part-based decision procedures. Starting by a certain product part, a decision should be made every time to choose which of the remaining unassembled parts is to be assembled next.

These difficulties make the introduced planners highly sensitive to any increase in the number of product parts. A new approach is introduced in (Sebaaly and Fujimoto, 1996) that overcomes these difficulties by applying genetic algorithms. A new mapping is introduced to transform any random sequence into a feasible one satisfying the assembly rules and constraints, which are assumed available or can easily be extracted from the product design $\mathrm{CAD}$ files. This mapping paves the way to GA application to the assembly planning problem, thus overcoming many disadvantages of the previous exact-solution part-based-decision algorithms. A sequence population is chosen at random. The introduced mapping is applied to transform it into a population of feasible sequences. The fitness function of every sequence in the feasible population is then calculated according to some assembly evaluations and constraints in which several criteria such as manipulability, directionality, stability and cost are embedded. After that, genetic operators are applied and a new generation of random sequences is produced. This procedure is repeated until a termination criterion is met. Although it overcomes the difficulties of many existing algorithms, this approach still need to be extended in two directions: 1- to become able to deal with more than one product at the same time. 2to take into consideration the effects of cascading the assembly operations of one or more products on a set of machines. The following sections constitute one of the earliest extensions in these directions. The input planning data used in this paper are a set of assembly rules and constraints, and a set of assembly evaluations and constraints. The derivation of these sets from the product design CAD files is beyond the scope of this paper. 


\section{ASSEMBLY VS. PROCESSING SCHEDULING}

The simplest general statement of the scheduling problem, is how to find the optimal or best schedule that assign a given set of jobs to a set of available machines or servers, according to some queuing criteria, such as the client or product timein-process and/or waiting time, machines or servers idle time, and system or line balancing. This definition holds for several types of scheduling problems, such as traffic lighting, social services centres, and more specifically in industrial processes.

Industrial processes however, are divided into two phases, parts processing and assembly, each with different characteristics and constraints. Consequently, the corresponding scheduling problems are of different natures.

In parts processing for instance, a limited number of jobs is to be performed in a fixed order on a given material to fabricate a certain product part (Fujimoto, et al., 1994). Moreover, jobs of a certain part have no order relations or constraints whatsoever with the jobs of any other part. This part independence reduces the complexity of the scheduling problem since only few job ordering constraints should be satisfied, regardless of the number of parts to be manufactured.

In assembly processes on the other hand, one or more jobs should be performed to assemble two parts or subassemblies together, but the order in which parts are assembled is almost fixed (Lucertini, et al., 1994). Consequently, almost all jobs required to assemble a certain product are somehow related with a large set of ordering constraints. As a result, if a certain sequence order results in weak scheduling performances such as bottlenecks or large idle or waiting times, any changes proposed by the scheduling optimisation algorithm to overcome this weakness might lead to unfeasible subassemblies. Such cases are largely encountered in job-shop assembly environments, where one machine performs more than one job at different time intervals. The problem becomes even more difficult if more than one product are to be assembled by the same machines.

Another point that makes the assembly scheduling problem much more complicated than the manufacturing scheduling problem, is that the processing time for the same assembly job might vary according to the job order in the assembly sequence, while the manufacturing job processing time is always fixed. Consequently, different assembly sequences not only have different planning evaluations, but also different jobs processing times that leads to different scheduling evaluations. As a result, the traditional way of dealing with assembly planning and scheduling separately, by first generating the best plan or sequence, and then find the corresponding best schedule might not lead to a good solution. Instead, integrating both procedures would ensure the generation of the best sequence/schedule combination, although it might not contain the best schedule and/or the best sequence. 


\section{$\frac{\text { Product } 1}{R 11, R 12, R 13} \quad \frac{\text { Product } 2}{R 21, R 22, R 23, R 24} \quad \frac{\text { Product 3 }}{R_{31}, R_{32, R 33}}$ \\ Representation:}

$R 31, R 23, R 21, R 11, R$ 13,R22,R32,R24,R12,R33

Figure 1: Individual Representation.

A rather important difference between assembly scheduling and parts processing scheduling is that in the latter, the number and order of jobs needed to fabricate a certain part are fixed, while in assembly, both the number and order of jobs needed to connect a certain part differ from one sequence to another.

\section{MULTI-PRODUCT JOB-SHOP ASSEMBLY}

This section provides a basic description of the multi-product job-shop assembly. In job-shop environment, $m$ jobs should be performed by $n$ machines, where $m>n$. Usually, one machine performs a family of similar jobs. It is more flexible than the flow-shop environment, where only one job is allocated to each machine $(m=n)$. Assembly job-shop environments in particular are very complicated, due to the complicated precedence relations between almost all jobs that are performed by different machines. As a result, jobs to complete a certain connection cannot be performed before the jobs of its preceding connection finish, even if the corresponding machine is idle. The fact that all connections are ordered in a certain manner increases the occurrence probability of unappreciated behaviours. This fact is even more severe in systems that assemble more than one product simultaneously. In this paper, the multi-product job-shop assembly environment is considered, with the restriction that one machine can perform a family of operations, but each operation can only be performed by one machine.

\section{ASSEMBLY PLANNING AND SCHEDULING INTEGRATION}

\subsection{Sequence/Schedule Representation}

The representation of individuals considered to undergo the genetic operations is a sequence of all existing rules of every product at hand, arranged in a random sequence. For instance, for 3 products having 3,4 and 3 assembly rules respectively, the individual sequence representation consists of 10 rules arranged in a certain order as shown in figure 1. This representation contains both the assembly sequence for each product, and the overall schedule for all the products. For example in figure 1, product 1 is assembled in the order R11, R13 and R12. 


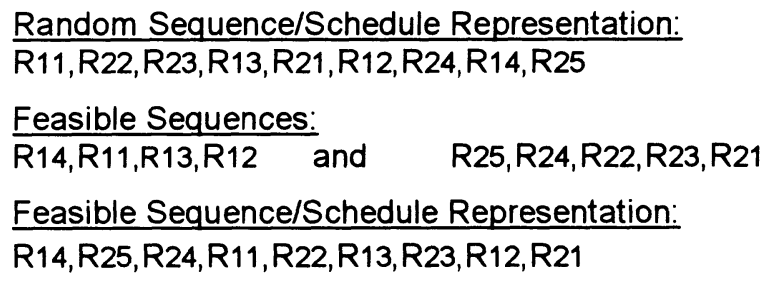

Figure 2: Repairing Sequence/Schedule Individuals.

However, R11 is performed after R31, R23 and R21. The schedule of these connections depends on both the number of machines and the processing time of each connection. The rules in a random individual are ordered in a random manner that might not respect the precedence constraints of every product. A random unfeasible individual is transformed into a feasible individual by repairing the rules order of products with violated constraints.

\subsection{Repairing Unfeasible Sequences}

Sequence feasibility is decided by the assembly rules and precedence constraints. Since the rules sequence is chosen to be the individual representation, then the assembly rules are for sure satisfied. What remains is to satisfy the assembly constraints. These constraints can be represented as precedence constraints between two rules. The precedence constraint $R 1<R 2$ means that rule 1 should precede rule 2. It follows that if this constraint is violated in a certain sequence, i.e. R2 is preceding $R 1$, it can be repaired by moving $R 2$ to the position just before $R 1$ in the sequence representation. This procedure is repeated for all violated constraints in a certain product, until the sequence becomes feasible.

In the sequence/schedule representation, where more than one product are represented, the rules of each product are taken out of their positions, repaired outside to represent a feasible sequence, and then returned to the same positions. For example in figure 2, the random sequence of Product 1 R11,R13,R12,R14 repaired to become $R 14, R 11, R 13, R 12$ and then returned back to the original positions as follows: R14 to position 1 in place of R11, R11 to position 4 in place of R13, etc. Product 2 is repaired and returned back in a similar manner.

\subsection{Multi-objective Fitness Function}

The fitness function is considered to be the reciprocal of the overall evaluation function. The overall evaluation function is composed of a linear combination of the planning and scheduling evaluation functions with constant coefficients, formulated as follows: 
Parent 1: R11, R12, R21, R22, R23
Parent 2: R21, R23, R22, R12 R11
Crossover Sites
Child 1: R11, R23, R22, R21, R12

Child 2: R22, R12, R21, R23, R11

Figure 3: Crossover Example

where $\varepsilon O V R$ : is the overall evaluation.

$\varepsilon P$ : is the total planning evaluation.

$\varepsilon T I P$ : is the time-in-process evaluation.

$\varepsilon I T:$ is the idle time evaluation.

$\alpha, \beta$ and $\gamma$ are constant coefficients.

\subsection{Genetic Operators}

\section{Reproduction}

The reproduction operator is applied to a population of chromosomes (Goldberg, 1989). Every chromosome should be a feasible sequence in the assembly case, in order to be evaluated. It is represented by the sequence/schedule representation, showing the rules sequences of the products at hand, with their execution order. After the fitness function of each individual in the population is calculated as described above, the traditional biased-roulette method (Goldberg, 1989)is applied to the population to perform the selection of the fittest and construct the mating pool.

\section{Crossover}

The crossover operator applied is an extension of the traditional two points crossover, with some additional restrictions to meet the requirements of the assembly problem. Two crossover sites are randomly chosen for the two mating parents. The rules of parent 1 and parent 2 are exchanged between these sites. However, while exchanging the rules, a replacement procedure should be done as follows to avoid duplications (refer to figure 3): Assume we are replacing R12 at position 2 of parent 1 by rule R23 from parent 2 . But R23 already exists at position 5 . To avoid duplication, we replace the already existing R23 at position 5 by the replaced rule $R 12$ of position 2 . Therefore, in the child1, R23 will be in position2 while R12 in position5.

\section{Mutation}

Two mutation operators is introduced to maintain complete sequences. The first randomly selects two rules their positions are exchanged. The other randomly selects a rule and flips its order. 


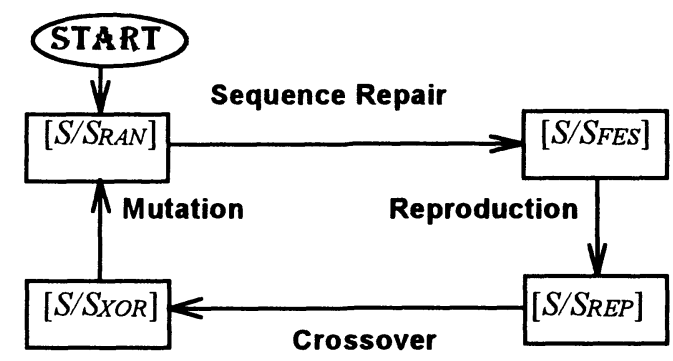

Figu re 4: GASS Schematic Diagram.

\section{THE OVERALL ALGORITHM}

The inputs of the overall algorithm are: (1) A set of rules and precedence constraints describing the product, extracted from the its CAD design files. (2) A set of evaluation assignments and constraints evaluating every rule according to its enclosed assembly operations. (3) A set of machine/job assignments classifying the job families and their corresponding machines. (4) A set of job processing times, showing the processing time for every job/operation, and the related constraints.

The detailed derivation of these sets is beyond the scope of this paper. The overall algorithm GASS is shown in figure 4. It proceeds as follows:

- Step 1: Starting by the input sets, generate a random population [S/SRAN] of $M P O P$ sequence/schedule individuals, where $M P O P$ is the number of individuals in the population.

- Step 2: Apply the repairing procedure to transform the population [S/SRAN] into a feasible population $[S / S F E S]$.

- Step 3: Calculate the fitness function of every individual as described above, and apply the reproduction operator to prepare the mating pool $[S / S R E P]$.

- Step 4: Randomly choose a mate for every individual and apply the crossover operator.

- Step 5: Apply the mutation operators. The result will be a new generation of the random population [S/SRAN].

- Step 6: Repeat steps 3 through 5 until a termination criterion or an acceptable solution is reached.

The method adopted in this paper to designate the result of GASS is the bestso-far-individual, which designates the best individual obtained in any generation of the population during the run (Goldberg, 1989).

The reparation of the unfeasible individuals can be seen as a mapping that drives any sequence in the entire search space into the set of feasible solutions. The whole procedure can then be thought of as follows: The whole search space is clustered into families of solutions, each having only one feasible sequence/schedule 

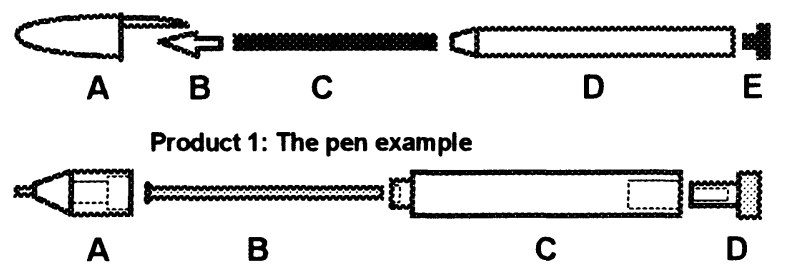

Product 2: The pencil example

Figure 5: A Prototype Example.

individual. A random population of individuals from random families are chosen to undergo the genetic operations. Each random individual asks his family representative -if he is not the representative himself- to represent him in these operations. The new generated population is of stochastic nature, i.e. might include ordinary family members -not representatives-. The same procedure is repeated, where the new corresponding families will send their representatives to be the next feasible generation to undergo the genetic operations, and so on.

When the run is terminated, the obtained best sequence/schedule represents the best sequence to assemble the products taking into account the effects of cascading the operations in the resulting order, and the best schedule that distributes jobs on appropriate machines, without altering the physical product constraints.

\section{AN ILLUSTRATIVE EXAMPLE}

A prototype example is constructed and solved to illustrate this methodology. It consists of two products that should be assembled by two machines. The products consisting of a pen and a pencil are shown in figure 5. The input sets of the algorithm are derived to be as follows:

\section{Product 1}

- Assembly rules:

$\mathrm{AD}, \mathrm{BC}, \mathrm{BD}, \mathrm{DE}$.

- Assembly Constraints:

$\mathrm{BC}<\mathrm{BD}$ and $\mathrm{BD}<\mathrm{DA}$.

- Evaluations:

$|\mathrm{CB}|=4 ; \quad|\mathrm{BC}|=12 ;$ (tight insertion)

$|\mathrm{DB}|=3 ; \quad|\mathrm{BD}|=9$; (insertion)

$|\mathrm{DA}|=2 ; \quad|\mathrm{AD}|=6$; (loose insertion)

$|\mathrm{DE}|=3 ; \quad|\mathrm{ED}|=9$; (insertion)

Evaluation Constraints: $\quad$ If $\mathrm{BC}<\mathrm{BD}$ then $|\mathrm{DB}|=6$ and $|\mathrm{BD}|=18$

- Machine Assignments and processing times are shown in Table 1.

Processing time constraints: If $\mathrm{BC}<\mathrm{BD}$ then $\mathrm{BD} \rightarrow 4$

\section{Product 2}

- Assembly rules: $\quad \mathrm{AB}, \mathrm{AC}, \mathrm{BC}, \mathrm{BD}, \mathrm{CD}$. 
Table 1 Machine Assignments and Processing time of product 1:

\begin{tabular}{llc}
\hline Rule & Machine: & Time: \\
\hline$-\mathrm{AD}$ & $\mathrm{M} 1$ & 1 \\
\hline$-\mathrm{BC}$ & $\mathrm{M} 1$ & 3 \\
\hline$-\mathrm{DE}$ & $\mathrm{M} 2$ & 2 \\
$-\mathrm{BD}$ & $\mathrm{M} 2$ & 2 \\
\hline
\end{tabular}

Table 2 Machine Assignments and Processing time of product 2:

\begin{tabular}{llc}
\hline Rule & Machine & Time \\
\hline$-\mathrm{AB}$ & $\mathrm{M} 1$ & 1 \\
$-\mathrm{AC}$ & $\mathrm{M} 1$ & 4 \\
\hline$-\mathrm{BC}$ & $\mathrm{M} 2$ & 2 \\
$-\mathrm{BD}$ & $\mathrm{M} 2$ & 3 \\
\hline$-\mathrm{CD}$ & $\mathrm{M} 2$ & 2 \\
\hline
\end{tabular}

- Assembly Constraints:

$\mathrm{BC} \leq \mathrm{AC} ; \mathrm{BC} \leq \mathrm{BD}$ and $\mathrm{AB} \leq \mathrm{AC}$.

- Evaluations:

$$
\begin{array}{ll}
|\mathrm{BA}|=1 ; & |\mathrm{AB}|=3 ; \text { (contact) } \\
|\mathrm{CB}|=3 ; & |\mathrm{BC}|=9 ; \text { (insertion) } \\
|\mathrm{CA}|=5 ; & |\mathrm{AC}|=15 ; \text { (rotation) } \\
|\mathrm{CD}|=2 ; & |\mathrm{DC}|=6 ; \text { (loose insertion) } \\
|\mathrm{BD}|=4 ; & |\mathrm{DB}|=12 ; \text { (tight insertion) }
\end{array}
$$

- Evaluation Constraints: If $\mathrm{AB}<\mathrm{BC}$ then $|\mathrm{CB}|=9$ and $|\mathrm{BC}|=27$

$$
\text { If } \mathrm{CD}<\mathrm{BC} \text { then }|\mathrm{CB}|=6 \text { and }|\mathrm{BC}|=18
$$

If $\mathrm{BC}<\mathrm{BD}$ and $\mathrm{BD}<\mathrm{AC}$ then $|\mathrm{BD}|=10$ and $|\mathrm{BC}|=30$

- Machine Assignments and processing times are shown in Table 2.

- Processing time constraints: If $\mathrm{CD}<\mathrm{BC}$ then $\mathrm{BD} \rightarrow 1$

$$
\text { If } \mathrm{BC}<\mathrm{BD} \text { and } \mathrm{BD}<\mathrm{AC} \text { then } \mathrm{BD} \rightarrow 2
$$

In the evaluation assignments, it is assumed that moving the larger part and assembling it to the smaller one (e.g. C to B) is evaluated three times more than moving the small part and assembling it to the large one (e.g. CB).

The proposed algorithm is applied with appropriate operator rates for 100 generations with a population of 10 individuals. The planning, scheduling and overall evaluations are shown in figure 6 (Ep, Es and Eovr respectively). It can be seen that the sequence resulting in the best schedule $(E s=12)$ in generations 3 to 26 has a high planning evaluation of 19.5 , and results in an overall evaluation of 31.5 , while the best sequence/schedule has an over all evaluation of 26.5 , with $E p=13.5$ and $E s=13$, which is not the best schedule. Samples of the sequence/schedules obtained at different generations and the corresponding sequences and schedules are shown in figures 7 and 8 respectively. The fact that the best schedule does not correspond to the best sequence, and vice verse, is imposed by the highly constrained nature of the assembly scheduling problem. 


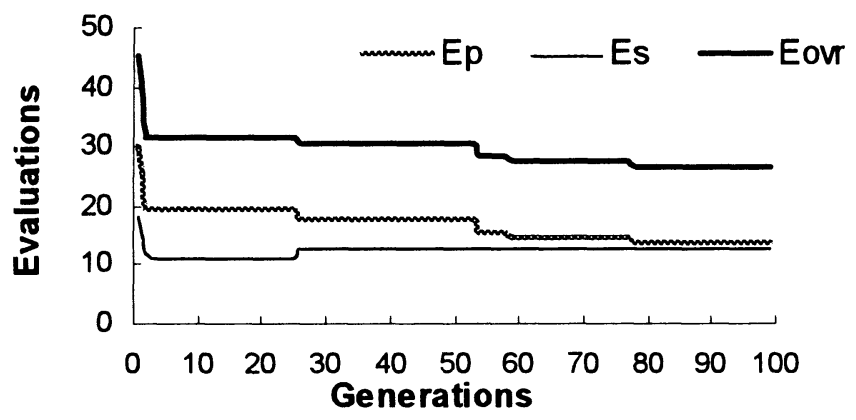

Figure 6: Fitness vs. Generation Graph.

$$
\begin{aligned}
& \text { 1) } E p=19.5, E s=12, \text { Eovr }=31.5 \text {, Gener }:: 3 \\
& \text { *Sequence/Schedule: } \\
& \mathrm{CB} 2, \mathrm{CB} 1, \mathrm{CD} 2, \mathrm{BA} 2, \mathrm{CA} 2, \mathrm{DB} 1, \mathrm{DE} 1, \mathrm{DB} 2, \mathrm{AD} 1 \\
& \text { 2) } E p=17.5, E s=13, \text { Eovr }=30.5, \text { Gener }: 28 \\
& \text { * Sequence/Schedule: } \\
& \mathrm{CB} 1, \mathrm{BC} 2, \mathrm{AB} 2, \mathrm{CA} 2, \mathrm{DE} 1, \mathrm{DB} 1, \mathrm{DA} 1, \mathrm{CD} 2, \mathrm{BD} 2 \\
& \text { 3) } E p=13.5, E s=13, \text { Eovr }=26.5 \text {, Gener } .: 80 \\
& \text { *Sequence/Schedule: } \\
& \mathrm{CB} 1, \mathrm{CB} 2, \mathrm{BA} 2, \mathrm{DE} 1, \mathrm{CA} 2, \mathrm{DB} 1, \mathrm{CD} 2, \mathrm{DA} 1, \mathrm{BD} 2
\end{aligned}
$$

Figure 7: Sample Sequence/Schedule Results.

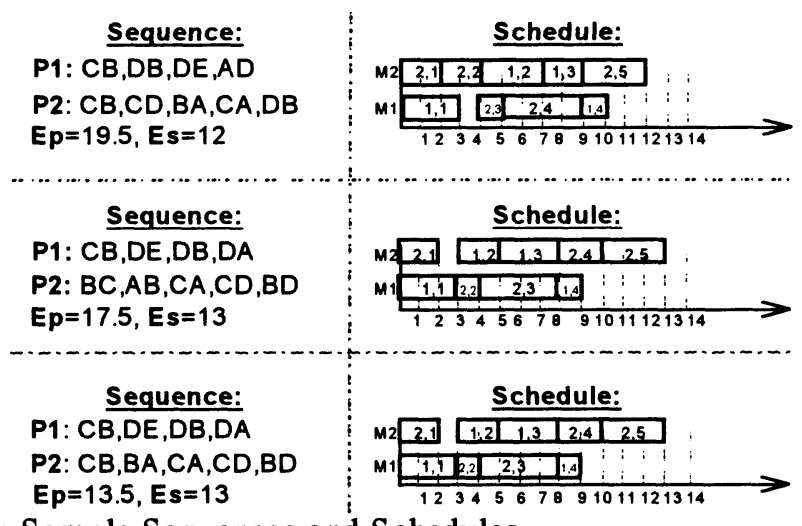

Figure 8: Sample Sequences and Schedules.

\section{CONCLUSION}

This paper addresses the problem of integrated assembly planning and scheduling based on genetic algorithms. A new sequence/schedule representation is introduced as a solution individual. Starting by a random population of individuals, unfeasible 
individuals are repaired and then ordinary GA operators are applied. This procedure is repeated until some stopping criteria are encountered. As a result, a best sequence and schedule are generated simultaneously, thus overcoming the difficulties encountered when planning and scheduling are dealt with separately.

\section{REFERENCES}

Fujimoto, H., Yasuda, K. and Iwahasi, K. (1994), Simulation Analysis of Design Rule Sets for Multilevel Production Scheduling in FMS, in 'Proceedings of the Japan-USA Symposium on Flexible Automation', pp. 811-814.

Goldberg, D. (1989), Genetic Algorithms in Search, Optimization, and Machine Learning. Addison-Wesley Pub. Inc., pp.12-41.

Lucertini, M., Pacciarelli, D. and Pacifici, A. (1994), Layout Constraints inAssembly Problems, in 'Proceedings of the Japan-USA Symposium on Flexible Automation', pp. 765-768.

$\mathrm{Pu}$, P. (1992), An Assembly Sequence Generation Algorithm using Case-based Search Techniques". in 'Proceedings of the IEEE International Conference on Robotics and Automation', pp.2425-2430.

Sebaaly, M. and Fujimoto, H. (1996) Automatic Assembly Planning Based on Genetic Algorithms, in 'Proceedings of the IFAC'96 World Congress', Vol. B, pp. 43.

Wolter, J., Chakrabarty, S. and Tsao, J. (1992), Mating Constraint Languages for Assembly Sequence Planning, in 'Proceedings of the IEEE International Conference on Robotics and Automation', pp. 2367-2374.

\section{BIOGRAPHY}

Milad Fares Sebaaly received his B.E. and M.E. in Electrical Engineering from the American University of Beirut, Beirut, Lebanon, in 1991 and 1994 respectively. He is currently a Doctor Student at the Mechanical Engineering Department of Nagoya Institute of Technology, Nagoya, Japan. His research interests mainly include factory automation, soft computing, assembly, robotics, concurrent engineering.

Hideo Fujimoto received his B.S. degree from Nagoya University in 1970, his M.S. degree from Nagoya Institute of Technology in 1972, and his Doctor of Engineering degree from Nagoya University in 1980, all in Mechanical Engineering. He was a visiting Professor at Technischen Hotchschule Aachen, West Germany, and at MIT, USA from 1985 to 1986 . He is currently a professor at the Mechanical Engineering Department of Nagoya Institute of Technology, Nagoya, Japan. He is the reviewer and editor of several international journals. His research interests mainly include: CIM, automation, robotic systems, virtual reality, human interface, medical mechatronics, etc. 